\title{
In Vitro Evaluation of Antimicrobial Effect of Extracts of Medicinal Plant's Leaves
}

Ale Zehra*, Syed Baqir Shyum Naqvi and Syeda Qadam Ali

Department of Pharmaceutics, Faculty of Pharmacy, University of Karachi, Pakistan

\begin{abstract}
In ancient and modern epoch, aerial parts of herbal plants have been broadly used for the treatment of primary health care and variety of ailment across the world depends on geographical cultivation. Now a day's researcher focuses their attention to explore the plants having broad spectrum therapeutic activity. On the basis of medicinal plant activity surveillance, the core goal step of current study is to evaluate the susceptible antimicrobial activity of crude $50 \%$ hydro alcoholic extract of leaves of different folk medicinal plant i.e Ocimum basilicum L., Cymbopogon citratus (DC.) Stapf., Olea europaea L., Eucalyptus camaldulensis Dehnh. against different clinical isolates (Enterococcus faecalis, Escherichia coli, Klebsiella pneumoniae, Bacillus subtilis, Pseudomonas aeruginosa, Staphylococcus aureus, Staphylococcu epidermidis, Salmonella typhi and Candida albican) of microbial disease so as to trip up on the other alternatives and overcome the upcoming era of increasing microbial resistance. These isolates were collected from different hospitals and pathological laboratories of Karachi, Pakistan. Extract were obtain by soaking the leaves in $50 \%$ methanol and then vacuum dried through Rotavapor while the antimicrobial activity were evaluated by well diffusion method. Overall outcome of current study endorse that among selected herbs, Olea europea possessed broad spectrum antimicrobial activity. Further investigation is needed to develop formulation from the same plant. It's timely need to explore the antimicrobial activity of other herbs also.
\end{abstract}

Keywords: Clinical isolates; Antimicrobial herbs; Ocimum basilicum; Cymbopogon citrate; Olea europaea; Eucalyptus camaldulensis

\section{Introduction}

Microbes are microscopic organism that includes any unicellular, cell bunches, or a cellular [1], found nearly everywhere in the taxonomic society of life on the sphere. In modern epoch, medical science along with other different challenges also stand in front of a challenge of escalating microbial resistance against different antibiotic [2] due to which now at present, researchers focuses on antimicrobial activity of traditional plants to overcome the microbial resistant pattern [3-7] however most of the active compound are present in these plants that can be used globally for different health care purposes [8,9]. Microbes can develop beneficial endosymbiotic association with other organisms in spite of causing of many contagious infections. These microbes include pathogenic bacteria (asplague, tuberculosis, and anthrax), protozoa (malaria, sleeping sickness, toxoplasmosis), fungi (ringworm, candidiasis, histoplasmosis). Skin floras are habitually non-pathogenic or mutualistic microorganisms (mostly 1000 species of bacteria) exist on skin, mostly on superficial or upper layers of the epidermis and hair follicles [10-12]. The non-pathogenic bacteria can deal with avoiding transitory pathogenic organisms from colonizing the skin superficial. Though, the occupant organism on skin can cause infection and enter the blood system producing life-threatening infections mainly in immune compromise person [13].

Different aerial part of plant has been used since ancient time either extracted raw compound or make a paste [14]. Although several plant species have been evaluated as a best choice for antimicrobial activity [15-18]. As these plants contain such an essential component which is consider to be having an antimicrobial [19-21].

Earliest investigation specifies that $S$. epidermidis and Propionibacterium is globally resided inside pretentious acne vulgaris [22]. Candida albican when invade on superficial mucous membranes, yields a white and cheesy plaque that is roughly adhere on the mucosal outward, called lesion or oral thrush which is generally painless till the plaque is indecisive and the raw, weeping, mucosal superficial surface is wide-open. Oral thrush usually occurs as single or various scruffy white blotcheson on the mucosal surfaces like tongue and plates. Similary fungal infection of vagina occurs by vaginal candidiasis which produces a thick, curd-like release, itching and burning of the vulva. Multiple ranges of self-limiting fatal infections in humans are caused by $S$. aureus which is an opportunistic pathogen. Usually neonates and young children have scale skin syndrome which is due to exfoliative toxins produce on epidermis [23]. Similary blisters, pimples, boils, impetigo, folliculitis and other skin disorders like abscesses and skin loss are due to exfoliative toxin produce by Staphylococcus species [2326]. Immuno suppressive people have necrotizing fasciltis which is due to $S$. aureus though this condition is rare [27].

$P$. aeruginosa can also spontaneously colonize on open burn lesions, causing infections, carbuncles, sepsis, edema staining of unburned skin at wound boundaries, green pigmentation of subcutaneous fat. Klebsiella spp. has been identified as important common pathogens for wound infections of $2 \%-4 \%[28,29]$.

During present study, following 04 plants namely, Ocimum basilicum L., Cymbopogon citratus (DC.) Stapf., Olea europaea L., Eucalyptus camaldulensis Dehnh were used to evaluate their antimicrobial. These plants were selected to study because of their excessive usage in local and herbal medicines. Moreover, these plants are one of the native plants available in Pakistan. A number of researches have been already done on these plants but there isn't much

*Corresponding author: Ale Zehra, Department of Pharmaceutics, Faculty of Pharmacy, University of Karachi, Pakistan, Tel: 92-03462757106; E-mail: ale.zehra110@gmail.com

Received April 13, 2016; Accepted July 02, 2016; Published July 07, 2016

Citation: Zehra A, Naqvi SBS, Ali SQ (2016) In Vitro Evaluation of Antimicrobia Effect of Extracts of Medicinal Plant's Leaves. J Med Microb Diagn 5: 236 doi:10.4172/2161-0703.1000236

Copyright: ( 2016 Zehra A, et al. This is an open-access article distributed under the terms of the Creative Commons Attribution License, which permits unrestricted use, distribution, and reproduction in any medium, provided the original author and source are credited. 
work done in Pakistan. These plants are supposed to be very effective against different microbes. Therefore this study is made to compare the antimicrobial activities of selected plants with those done in other countries.

Sweet Basil is a communal name for the herb O. basilicum $\mathrm{L}$. belong to Lamiaceae family. Originated from India, Italian cuisine, Northeast Asian cuisine of Taiwan, Thailand, Cambodia, and Laos. The taste of leaves is similar to an anise with sweet smell often strong and pungent depending on the species and geographical cultivation [30]. Since ancient period of time, O. basilicum $\mathrm{L}$. has been used as culinary and medicinal herbs [31-33]. Various in vitro scientific studies proved that basil oil have strong antioxidant, and antimicrobial properties $[19,34,35]$

In Asian region, it is used for treating pimples on the face in siddha medicine. Large ingestion of the basil seeds quantities is harmful for the brain. Various studies in rats and mice proved that basil is carcinogen and teratogen due to presence of estragole [36].

Lemon grass is a common name of genus C. citratus (DC.) Stapf., containing 55 grass's species. Studies show that lemon grass oil has antifungal, antimicrobial, pesticide and preservative [37]. Due to having 65\%-85\% chemical component such as citral, citronellol, geranilol and myrcene as and an active ingredient, lemon grass has an antibacterial and pain reliever property $[21,38]$. It is cultivated at hot or warm area across the world most commonly origin at Philippines and Oceania. It is a tall perennial grass, broadly used in herbal drugs in Asian cuisine [21].

Leaf of the O. europaea L. is olive leaf. Since ancient period of time, the leaf of olive has been used medicinally. Globally the species of olive has cultivated around the world, Corsica, Greece, Crimea, Iraq, Israel, Italy, Palestine, Syria, Java, Africa, Portugal, Arabian Peninsula, Southern Asia and Bermuda.

The prime content of olive leaf is oleuropein [39] and hydroxytyrosol with certain polyphenols and flavonoids [26,40,41].

Olive leaf posse's good activity against bacterial and fungal microbes. Leaves of Olive play a vital role in health care system due to containing of certain phytochemical [42]. Olive leaf possessed antimicrobial activity on pathogens of food [43].

Olive leaf is generally used as antiviral, antibacterial and antifungal in many severe infections. Olive leaf not only used to treat infection caused by microbes but also used traditionally as hypolipidemic agent, anti-rheumatic, anti-inflammatory, antinociceptive, antipyretic, antidiuretic, calcium channel blocker property, normalize the blood pressure to relax the heart arteries, improve neurological deficit scores in stroke [26,44-51]. Olive leaf possessed antioxidant activity thus prevent biological system from free radical which is help ful in treating tumor and different cancre but it's antioxidant property is potent when used with other antioxidant [52- 57].

The River Red Gum tree is a common name of E. camaldulensis Dehnh. Leaves contain $77 \%$ cineol while certain amount of cuminal, phellandrene, aromadendren, valerylaldehyde, geraniol, cymene, phellandral, tannin,

\begin{tabular}{|c|c|c|}
\hline Study Herb & Scientific name & Specimen name \\
\hline Basil & Ocimum basilicum L. & Ob-12-07 \\
\hline Lemon grass & Cymbopogon citratus (DC.) Stapf. & Ob-12-08 \\
\hline Olive & Olea europaea L. & Ob-12-09 \\
\hline Red River Gum & Eucalyptus camaldulensis Dehnh. & Ob-12-10 \\
\hline
\end{tabular}

Table 1: Specification number. glucoside, catechol, and pyrocatechol. Leaves also have certain amount of flavonoids and sterols while annin is presence in bark [58]. It can be used as good choice for antibacterial formulation [59].

\section{Methodology}

\section{Collection of clinical isolates}

Pathogenic clinical isolates i.e. bacteria used in current study include E. faecalis, E. coli, K. pneumoniae, B. subtilis, P. aeruginosa, S. aureus, S. epidermidis, S. typhi while fungi includes C. albicans. Suitable strains of all these microorganisms were procured from different pathological and microbiological Laboratories of Karachi, Pakistan. The organism were inoculated for $48 \mathrm{~h}$ on stationary culture of nutrient agar slant at $37^{\circ} \mathrm{C}$ and then inoculated in Mueller Hinton Agar medium.

\section{Isolation and identification of organism}

Clinical isolates were identified on the basis of morphological, cultural and biochemical reactions.

\section{Collection of aerial parts of plants}

Following selected plants for current study i.e. O. basilicum L., C. citratus (DC.) Stapf., O. europaea L., E. camaldulensis Dehnh., were collected from different region of Pakistan. All studied plants were provided and identified by pharmacist Dr. Iqbal Azhar who is specialist in pharmacogonosy, Department of Pharmacognosy, Faculty of Pharmacy, University of Karachi, Pakistan. Following specification number $\left(^{*}\right)$ were provided for authenticity of the plants (genus with species) used in this study as shown in Table 1.

The leaves of studied plant were collected and washed with water for removal of any impurities then washed again but finally wash with distilled water and kept the leaves for dry.

\section{Preparation of extract}

\section{Soaking of leaves in solvent}

The leaves of studied plant wrapped in plain uncontaminated paper and placed under shaded area to allow it for complete dry. Each set of studied plant's leaves were weight up to $100 \mathrm{gm}$ which were then transfer to large sterile conical flask individually. The flask were filled with $2000 \mathrm{ml}$ of $50 \%$ hydro alcoholic solvent which comprises of 1000 $\mathrm{ml}$ of $50 \%$ methanol and $1000 \mathrm{ml}$ of distilled water in such a way so that leaves were completely dip. Each flask was labelled with its plant name and date. Each conical flask was closed air tightly and covered with aluminium foil then kept in laboratory for about 02-03 weeks on average room temperature i.e. $25^{\circ} \mathrm{C}-30^{\circ} \mathrm{C}$ as shown in Figure 1 .

\section{Extraction}

The solvents of each flask were filtered by passing through filter paper to collect apparent colored filtrate. Clear filtrate was shifted to round bottom flask $\left(\mathrm{Pyrex}^{\otimes}\right)$ for vacuum dried through rotavapor (Rotavapors Buchi model R-114 Assembly A) as shown in Figure 2. Passing through rota vapor, the hydro alcoholic solvent was evaporated and final product appeared in form of thick, dark coloured and viscous exatract of leaves as shown in Table 2 .

The viscous extract was then transferred in separate sterile air tight small jar as shown in Figure 3. These small jars were weighted and volume measure then labelled with plant's name which were then kept in refrigerator till further use as describe in Bacteria Genomes [11]. The procedure for preparing extract of leaf of studied plants was repeated on need basis for more extract. 
Citation: Zehra A, Naqvi SBS, Ali SQ (2016) In Vitro Evaluation of Antimicrobial Effect of Extracts of Medicinal Plant's Leaves. J Med Microb Diagn 5: 236. doi:10.4172/2161-0703.1000236

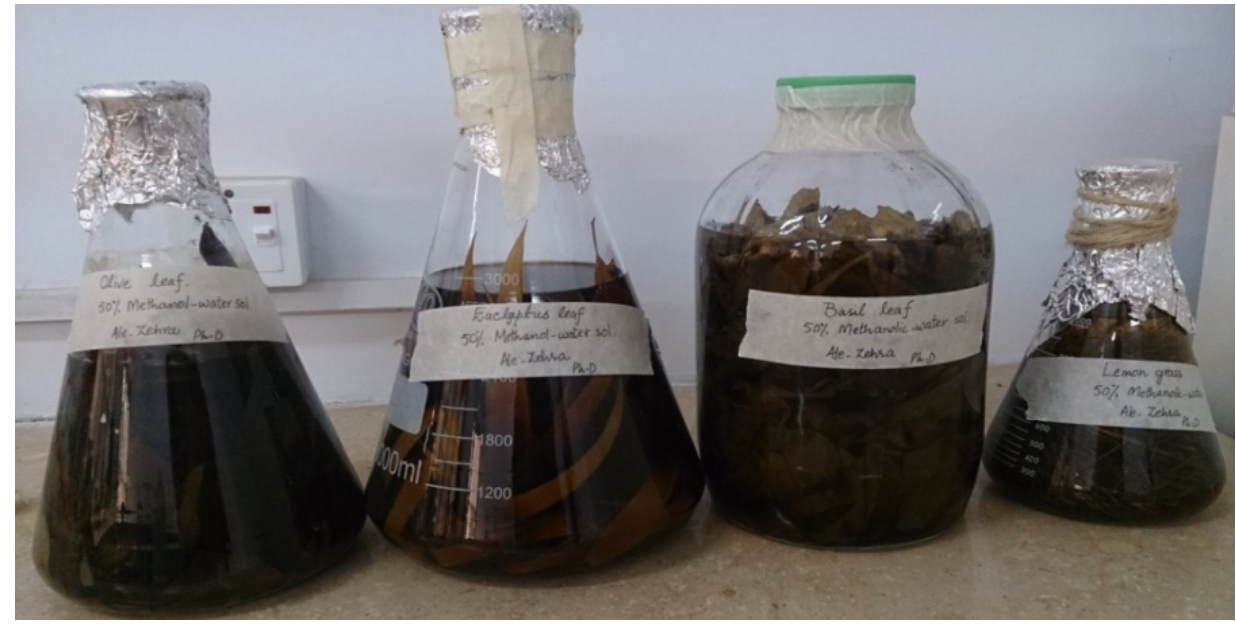

Figure 1: Areial part (Leaf) of plant soaked in hydro-alcoholic solvent for about 15 days

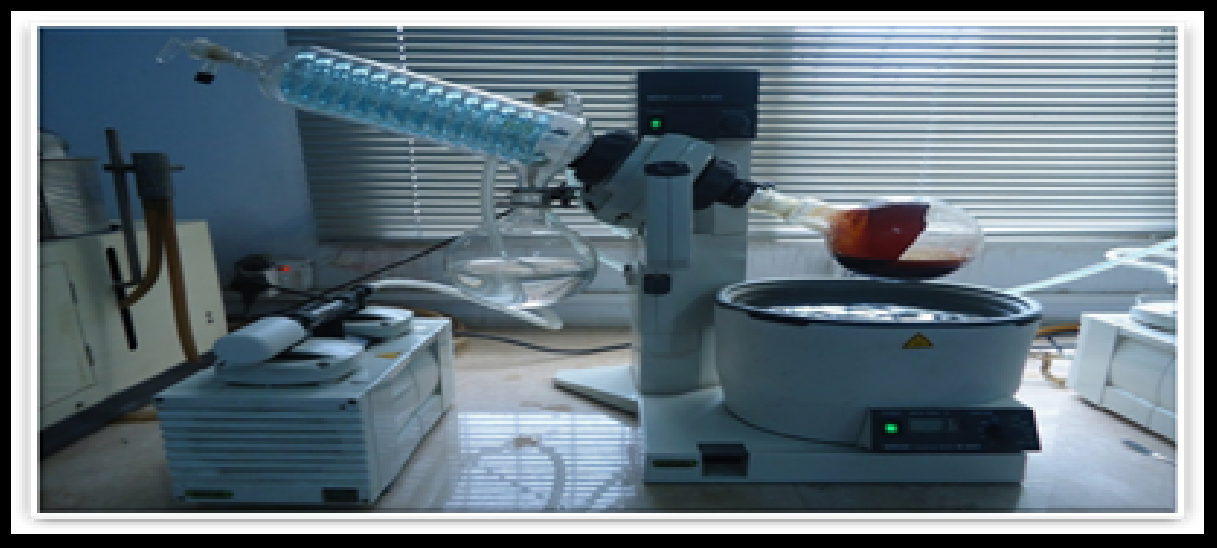

Figure 2: Rotavapor used during the study work showing Olea europaea L. extract to evaporate.

\begin{tabular}{|c|c|c|c|}
\hline Plants & Colour & Texture & Odour \\
\hline Ocimum basilicum & Dark brown & Sticky & Herbal sweet \\
\hline Cymbopogon citratus & Blackish brown & Thick paste & Slightly fishy \\
\hline Olea europaea & Dark brown & Sticky and Oily & Herbal sweet \\
\hline Eucalyptus camaldulensis & Reddish brown & Oily and sticky & Very pungent \\
\hline
\end{tabular}

Table 2: Unique features of extract of leaves of selected medicinal plants.

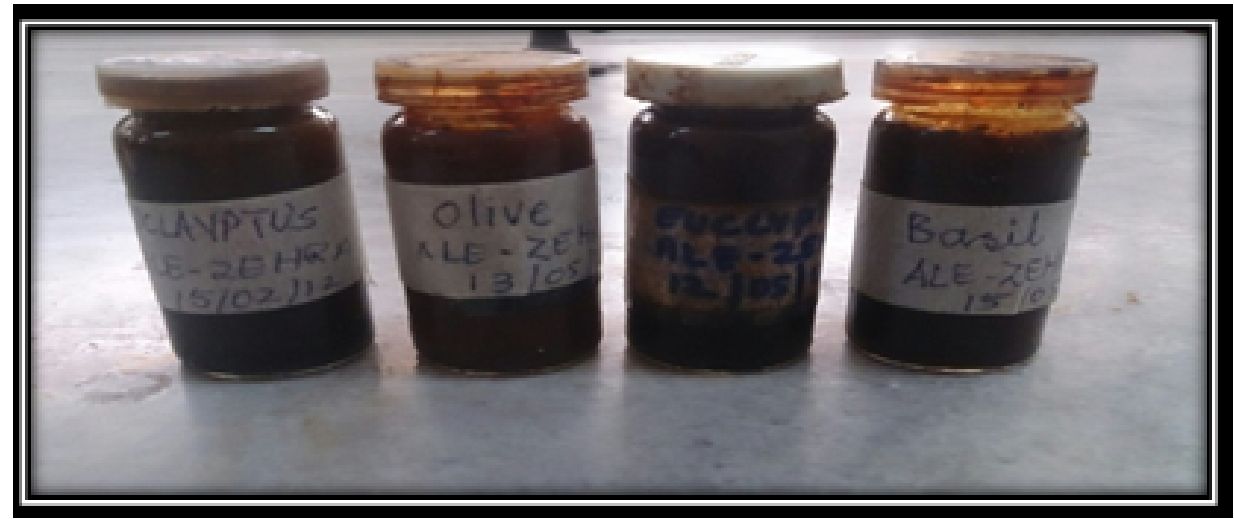

Figure 3: Viscous leaves extract (after evaporated from Rotavapor), collected in separate sterile small air tight labelled glass bottles. 


\section{Susceptibility testing}

Following steps were taken to check the susceptibility pattern of different clinical isolates against different plant extracts through agar well diffusion method $[60,61]$ and as describe by [62] while the test for positive control was done through disk diffusion method $[63,64]$.

\section{Preparation of inoculums}

a. Dip the applicator swab into broth culture.

b. By rotating swab against inside of tube, removed the excess fluids.

\section{Inoculation of agar}

a. Swab was streaked thoroughly on surface of the agar plate (150 mm Mueller-Hinton agar Petri dish).

b. Allowed the agar plate to keep for a period of 5-10 minutes to facilitate the broth absorbed into the agar.

\section{Development of well of extraction of studied plant leaves}

- Well were formed on different sides of agar plate through $1 \mathrm{~cm}$ diameter sterilized metal borer which were again sterile by keeping the metal borer punching side near flame for few seconds.

- The extracts of leaves were individually filled in a well in a quantity of $0.3 \mathrm{ml}$ which was previously labelled on back side of plate as shown in Figures 4-12.

- Used of $5 \mu \mathrm{g}$ Ciprofloxacin and $10 \mu \mathrm{g}$ Fluconazole disc as standard control for antibacterial and antifungal activity respectively.

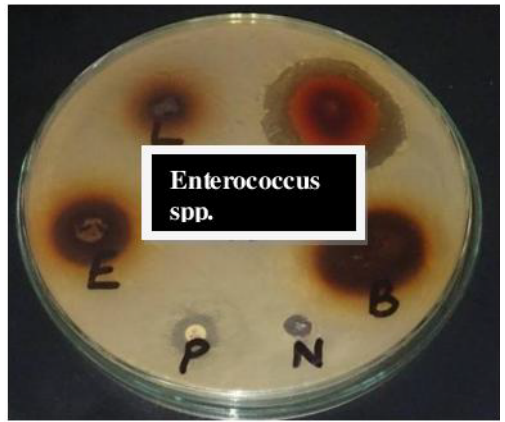

Figure 4: Agar plate showing susceptibillity of organisam through of inhibition against extract of studied medicinal plants, positive (ciprofloxacin) control and negative ( $50 \%$ hydroalcoholoic solvent) control.

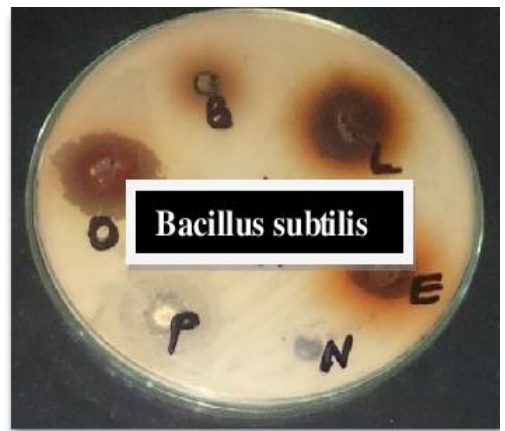

Figure 5: Agar plate showing susceptibillity of organism through zone of inhibition against extract of studied medicinal plants, positive (ciprofloxacin) and negative (50\% hydroalcoholic solvent) control.

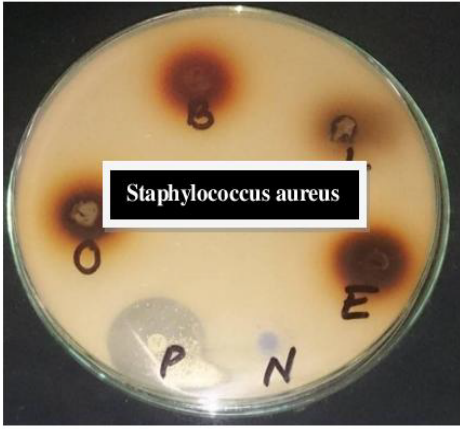

Figure 6: Agar plate showing susceptibillity of organisam through zone of inhibition against extract of studied medicinal plants, positive (ciprofloxacin) and negative (50\% hydroalcoholic solvent) control.

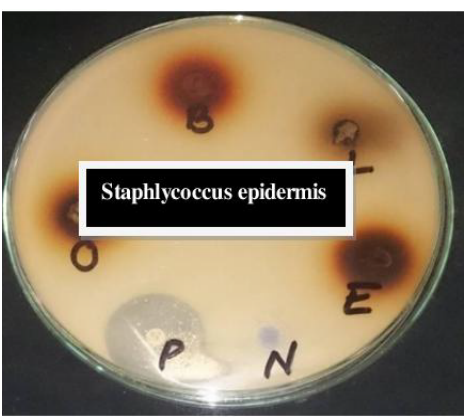

Figure 7: Agar plate showing susceptibillity of organisam through zone of inhibition against extract of studied medicinal plants, positive (ciprofloxacin) and negative ( $50 \%$ hydroalcoholic solvent) control.

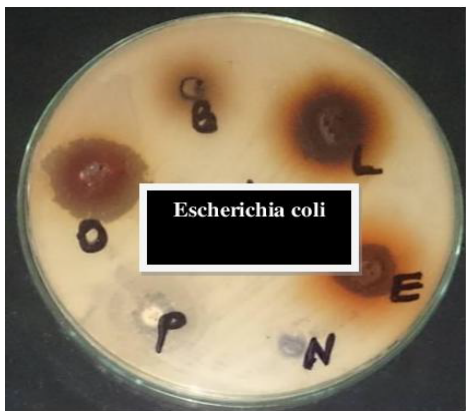

Figure 08: Agar plate showing susceptibillity of organisam through zone of inhibition against extract of studied medicinal plants, positive (ciprofloxacin) and negative (50\% hydroalcoholic solvent) control.

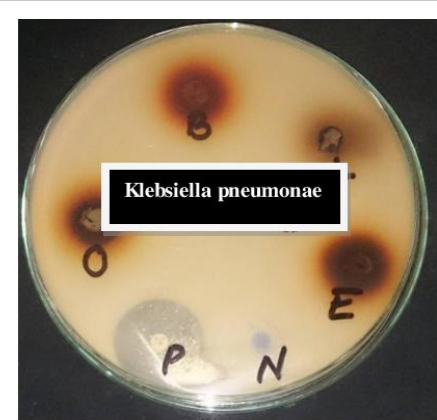

Figure 9: Agar plate showing susceptibillity of organisam through zone of inhibition against extract of studied medicinal plants, positive (ciprofloxacin) and negative $(50 \%$ hydroalcoholic solvent) control. 


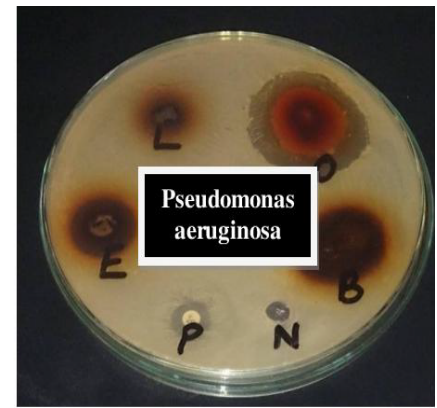

Figure 10: Agar plate showing susceptibillity of organisam through zone of inhibition against extract of studied medicinal plants, positive (ciprofloxacin) and negative $(50 \%$ hydroalcoholic solvent) control.

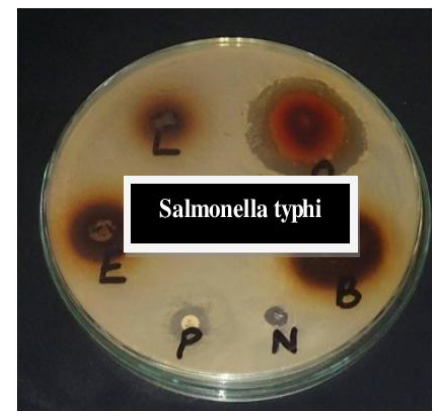

Figure 11: Agar plate showing susceptibillity of organisam through zone of inhibition against extract of studied medicinal plants, positive (ciprofloxacin) and negative $(50 \%$ hydroalcoholic solvent) control.

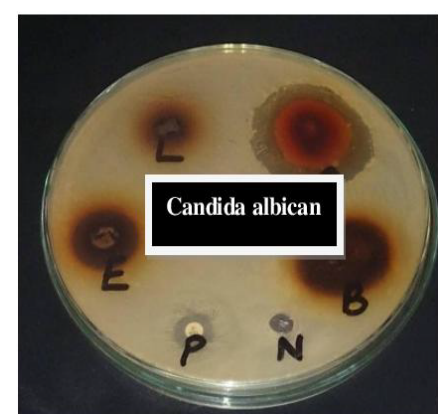

Figure 12: Agar plate showing susceptibillity of organisam through zone of inhibition against extract of studied medicinal plants, positive (fluconazole) and negative (50\% hydroalcoholic solvent) control.

- Similarly for negative control, six drop i.e. $0.3 \mathrm{ml}$ of pure hydro alcoholic solvent was used in current study.

\section{Incubation of plates}

a. The plates were incubated at $37^{\circ} \mathrm{C}$ for about 24 hours in incubator.

\section{Reading results}

a. With the help of caliper, zones of inhibition were measured in millimetres.

b. Record results

c. The result of disk diffusion method was carried out twenty times for each clinical isolates and mean of each zone of inhibition was used as a final result as shown in Tables 3-7.

\section{Interpretation of result}

The result of susceptibility testing was interpreted by method as describe by [6].

\section{Results and Discussion}

Studies to evaluate their specific antimicrobial properties have been done to negligible extent. The antimicrobial activity of most of the plant extracts were carried out by biological screening through utilizing a number of pathogenic as well as non-pathogenic microbes which is usually done by means of in vitro assay (disc diffusion and cylinder plate).

Current study was performed to evaluate the antimicrobial activity of four medicinal plants having traditional usage since ancient period of time namely: O. basilicum L. (Basil), C. citratus DC. Stapf. (Lemon Grass), O. europaea L. (Olive), E. camaldulensis Dehnh. (River Red Gum). Aerial part of selected plant for current study was leaves. Due to increase resistance pattern of microbe and reduction in treatment efficacy [65], it's timely need to explore the antimicrobial activity from herbal plant to make an antimicrobial formulation from it. Total 08 bacterial strains and 01 fungal strain were studied for the evaluation of sensitivity pattern in which $04 \mathrm{Gram}$ positive bacteria (E. faecalis, B. subtilis, S. aureus, S. epidermidis), 04 Gram negative bacteria (E. coli, K. pneumonae, P. aeruginosa, S. typhi) as shown in Tables 1-3. Fungi (candida albican) were selected for current study against 04 selected extract of studied medicinal plant as shown in Table 6.

Each bacterial and fungal strain were tested 20 times taken from different resources (Blood, Urine, Ear Pus) as shown in Tables 3-5 and its average mean were summarize as shown in Table 7.

Test for positive and negative control has been done by using a Ciprofloxacin $5 \mu \mathrm{g}$ disc for bacteria and fluconazole $10 \mu \mathrm{g}$ disc for fungal strain as an standard as shown in Table 6 while hydro alcoholic solvent used as a negative control.

The antibacterial assay was performed through agar well diffusion method $[60,61]$ as shown in Figures $4-11$ while the test for positive control was done through disk diffusion method [62-64] as shown in Figures 4-11.

The antifungal activity was screened by the method as describe in [62] on fungal strain, C. albican against extract of 04 studied medicinal plants as shown in Table 4.

\section{Olea europaea}

Extensive work has been carried out on medicinal activity of different aerial parts of O. europaea L. (olive) specially leaf part [66-72]. Besides having antimicrobial activity [73-75], olive leaf also showed a good efficacy as anti-inflammatory, antidiabetic, hypocholestrolemic, antiplatelate, analgesic [76-81] etc. Extract of olive leaf were used against 04 selected Gram positive bacteria, 04 Gram negative bacteria and 01 fungal strain.

Extract of olive leaf showed good activity as compare to other herbal extract on all microbes as shown in Table 7. In vitro good susceptibility effect of olive leaf extract was higher on S. aureus, E. feacalis, B. subtilitis, K pneumonae, S. typhi and C. albicans. Greater zone of inhibition was shown by C. albicans i.e. $25.6 \mathrm{~mm}$ as shown in Table 7 which is almost similar to zone of inhibition of fluconazole (broad spectrum antifungal) as shown in Table 6.

B. subtilitis plays a vital role in skin infection, also showed a good 
Citation: Zehra A, Naqvi SBS, Ali SQ (2016) In Vitro Evaluation of Antimicrobial Effect of Extracts of Medicinal Plant's Leaves. J Med Microb Diagn 5: 236. doi:10.4172/2161-0703.1000236

Page 6 of 14

\begin{tabular}{|c|c|c|c|c|c|}
\hline \multirow{2}{*}{ S.no } & \multicolumn{5}{|c|}{ Zone of inhibition ( $\mathrm{mm}$ ) of Gram positive bacteria } \\
\hline & Medicinal Plants & Staphylococcus aureus & Staphylococcus epidermidis & Bacillus subtilis & Enterococcus faecalis \\
\hline 1 & Ocimum basilicum & 18 & 11 & 21 & 17 \\
\hline 2 & O.basilicum & 15 & 0 & 20 & 15 \\
\hline 3 & O. basilicum & 20 & 0 & 21 & 15 \\
\hline 4 & O. basilicum & _- & 12 & 24 & - \\
\hline 5 & O. basilicum & 13 & 13 & 22 & 14 \\
\hline 6 & O. basilicum & 17 & 0 & 29 & 15 \\
\hline 7 & O. basilicum & 15 & 11 & 20 & 15 \\
\hline 8 & O. basilicum & 18 & 10 & 20 & 16 \\
\hline 9 & O. basilicum & - & 12 & 19 & - \\
\hline 10 & O. basilicum & 15 & 0 & 20 & 12 \\
\hline 11 & O. basilicum & 12 & 10 & 25 & 12 \\
\hline 12 & O. basilicum & 20 & 9 & 20 & 12 \\
\hline 13 & O. basilicum & 10 & 12 & 20 & 10 \\
\hline 14 & O. basilicum & 11 & 10 & - & 14 \\
\hline 15 & O.basilicum & - & 13 & 20 & - \\
\hline 16 & O.basilicum & 16 & 0 & 0 & 10 \\
\hline 17 & O. basilicum & 11 & 10 & 18 & 11 \\
\hline 18 & O. basilicum & 15 & 13 & 17 & 10 \\
\hline 19 & O. basilicum & 14 & 13 & 0 & 14 \\
\hline 20 & O. basilicum & 10 & - & 25 & 10 \\
\hline Mean & O. basilicum & 12.8 & 8 & 19 & 13 \\
\hline 1 & Cymbopogon citratus & 18 & 7 & 20 & 10 \\
\hline 2 & C. citratus & 15 & 8 & 18 & 9 \\
\hline 3 & C. citratus & 14 & 10 & 17 & 10 \\
\hline 4 & C. citratus & 11 & _ & 16 & - \\
\hline 5 & C. citratus & 14 & - & 15 & 14 \\
\hline 6 & C.citratus & 14 & 10 & - & 15 \\
\hline 7 & C.citratus & - & - & 10 & 15 \\
\hline 8 & C.citratus & 13 & 10 & _- & 16 \\
\hline 9 & C. citratus & 14 & 7 & 8 & - \\
\hline 10 & C. citratus & 15 & 10 & 14 & 12 \\
\hline 11 & C. citratus & 12 & 6 & 8 & 12 \\
\hline 12 & C. citratus & - & 5 & 15 & 12 \\
\hline 13 & C.citratus & 14 & 6 & 17 & 10 \\
\hline 14 & C. citratus & 16 & 7 & 10 & 14 \\
\hline 15 & C. citratus & 14 & 10 & 17 & - \\
\hline 16 & C. citratus & 15 & 14 & 11 & 10 \\
\hline 17 & C. citratus & 14 & _ & - & 11 \\
\hline 18 & C. citratus & 11 & 5 & 14 & 10 \\
\hline 19 & C. citratus & 14 & 6 & 10 & 14 \\
\hline 20 & C. citratus & 16 & 8 & 11 & 10 \\
\hline Mean & C. citratus & 14 & 8 & 13.5 & 12 \\
\hline 1 & Olea europaea & 30 & 10 & 20 & 24 \\
\hline 2 & O. europaea & 35 & 9 & 20 & 23 \\
\hline 3 & O. europaea & 20 & 10 & 20 & 21 \\
\hline 4 & O. europaea & 35 & *- & 18 & 26 \\
\hline 5 & O. europaea & 37 & 14 & 20 & 20 \\
\hline 6 & O. europaea & 23 & 15 & 23 & 23 \\
\hline 7 & O. europaea & 25 & 15 & 19 & 25 \\
\hline 8 & O. europaea & 25 & 16 & 19 & 30 \\
\hline 9 & O.europaea & 22 & - & 18 & 14 \\
\hline 10 & O.europaea & 24 & 12 & 19 & 23 \\
\hline 11 & O. europaea & 23 & 12 & 23 & 22 \\
\hline 12 & O. europaea & 21 & 12 & 21 & 30 \\
\hline 13 & O. europaea & 26 & 10 & 20 & 35 \\
\hline 14 & O. europaea & 20 & 14 & 20 & 20 \\
\hline 15 & O. europaea & 23 & - & 23 & 35 \\
\hline 16 & O. europaea & 25 & 10 & 25 & 37 \\
\hline 17 & O. europaea & 30 & 11 & 20 & 23 \\
\hline
\end{tabular}


Citation: Zehra A, Naqvi SBS, Ali SQ (2016) In Vitro Evaluation of Antimicrobial Effect of Extracts of Medicinal Plant's Leaves. J Med Microb Diagn 5: 236. doi:10.4172/2161-0703.1000236

Page 7 of 14

\begin{tabular}{|c|c|c|c|c|c|}
\hline 18 & O. europaea & 14 & 10 & 14 & 25 \\
\hline 19 & O. europaea & 23 & 14 & 23 & 25 \\
\hline 20 & O. europaea & 22 & 10 & 22 & 22 \\
\hline Mean & O. europaea & 25 & 12 & 20 & 25 \\
\hline 1 & Eucalyptus camaldulensis & 12 & 28 & - & 5 \\
\hline 2 & E. camaldulensis & 12 & 28 & 0 & 4 \\
\hline 3 & E. camaldulensis & 10 & 25 & 5 & 4 \\
\hline 4 & E. camaldulensis & 14 & 26 & - & _ \\
\hline 5 & E. camaldulensis & - & 20 & -- & - \\
\hline 6 & E. camaldulensis & 10 & 23 & 0 & 5 \\
\hline 7 & E. camaldulensis & 11 & 25 & 10 & _ \\
\hline 8 & E. camaldulensis & 10 & 30 & 12 & 5 \\
\hline 9 & E. camaldulensis & 14 & 14 & - & 7 \\
\hline 10 & E. camaldulensis & 10 & 23 & 10 & 5 \\
\hline 11 & E. camaldulensis & 9 & 22 & 9 & 4 \\
\hline 12 & E. camaldulensis & 10 & 23 & 12 & 5 \\
\hline 13 & E. camaldulensis & _ & 35 & 10 & 4 \\
\hline 14 & E. camaldulensis & 14 & 17 & 6 & 7 \\
\hline 15 & E. camaldulensis & 15 & 35 & - & 5 \\
\hline 16 & E. camaldulensis & 15 & 37 & 10 & 5 \\
\hline 17 & E. camaldulensis & 16 & 23 & 13 & _ \\
\hline 18 & E. camaldulensis & - & 25 & 13 & 5 \\
\hline 19 & E. camaldulensis & 12 & 19 & 11 & 6 \\
\hline 20 & E. camaldulensis & 12 & 22 & _ & 4 \\
\hline Mean & E.camaldulensis & 12 & 25 & & 5 \\
\hline
\end{tabular}

Table 3: Antimicrobial susceptibility of selected 04 Gram positive bacteria against $50 \%$ hydro alcoholic extract of leaves of medicinal plant.

\begin{tabular}{|c|c|c|c|c|c|}
\hline \multirow[t]{2}{*}{ S.no } & \multicolumn{5}{|c|}{ Zone of Inhibition (mm) of Gram Negative Bacteria } \\
\hline & Medicinal Plants & Escherichia coli & Klebsiella pneumonae & Pseudomonas aeruginosa & Salmonella typhi \\
\hline 1 & Ocimum basilicum & 3 & 10 & 5 & 25 \\
\hline 2 & O. basilicum & 4 & 9 & 4 & 24 \\
\hline 3 & O. basilicum & 4 & 10 & 3 & 25 \\
\hline 4 & O. basilicum & *_ & - & 5 & 26 \\
\hline 5 & O. basilicum & _ & 14 & 4 & 24 \\
\hline 6 & O. basilicum & 5 & 15 & _ & 23 \\
\hline 7 & O. basilicum & _ & 15 & 3 & 25 \\
\hline 8 & O. basilicum & 5 & 16 & 5 & 24 \\
\hline 9 & O. basilicum & 4 & _ & 4 & 25 \\
\hline 10 & O. basilicum & 3 & 12 & 7 & 28 \\
\hline 11 & O. basilicum & 4 & 12 & 5 & 24 \\
\hline 12 & O. basilicum & 5 & 12 & 6 & 27 \\
\hline 13 & O. basilicum & 4 & 10 & _ & 26 \\
\hline 14 & O. basilicum & 3 & 14 & - & 30 \\
\hline 15 & O. basilicum & 5 & - & 5 & 23 \\
\hline 16 & O. basilicum & 4 & 10 & _ & 25 \\
\hline 17 & O. basilicum & _ & 11 & 5 & 28 \\
\hline 18 & O. basilicum & 3 & 10 & 4 & 24 \\
\hline 19 & O. basilicum & 5 & 14 & 3 & 23 \\
\hline 20 & O. basilicum & 4 & 10 & 4 & 22 \\
\hline Mean & O. basilicum & 4 & 12 & 4.5 & 25 \\
\hline 1 & Cymbopogon citratus & 16 & 10 & 7 & 20 \\
\hline 2 & C. citratus & 14 & 9 & 8 & 21 \\
\hline 3 & C. citratus & 15 & 12 & 6 & 21 \\
\hline 4 & C. citratus & 14 & 10 & 7 & 19 \\
\hline 5 & C. citratus & 11 & 13 & _ & 20 \\
\hline 6 & C. citratus & 14 & - & 7 & 14 \\
\hline 7 & C. citratus & 16 & 10 & _ & 21 \\
\hline 8 & C. citratus & 18 & 13 & 5 & 18 \\
\hline 9 & C. citratus & 15 & 13 & 8 & 20 \\
\hline 10 & C. citratus & 14 & 11 & 8 & 27 \\
\hline 11 & C. citratus & 11 & - & 8 & 20 \\
\hline 12 & C. citratus & 14 & - & 5 & 18 \\
\hline 13 & C. citratus & 14 & 12 & 4 & 20 \\
\hline
\end{tabular}


Citation: Zehra A, Naqvi SBS, Ali SQ (2016) In Vitro Evaluation of Antimicrobial Effect of Extracts of Medicinal Plant's Leaves. J Med Microb Diagn 5: 236. doi:10.4172/2161-0703.1000236

Page 8 of 14

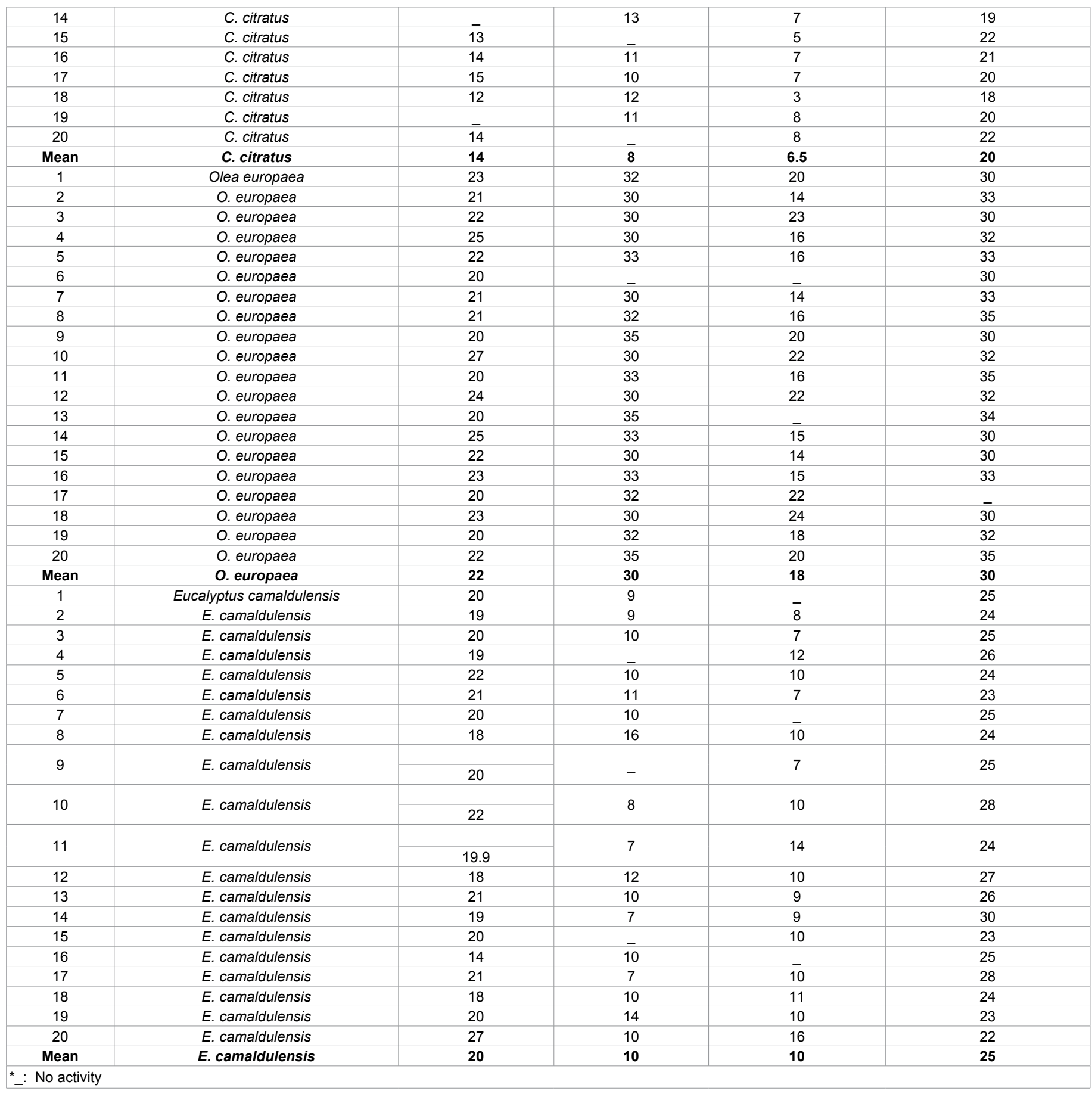

Table 4: Antimicrobial susceptibility of selected 04 Gram negative bacteria against $50 \%$ hydro alcoholic extract of leaves of medicinal plant.

\begin{tabular}{|c|c|c|c|c|c|c|c|c|c|c|c|c|c|c|c|c|c|c|c|c|c|c|}
\hline \multirow{3}{*}{$\begin{array}{l}\text { Medicinal } \\
\text { Plants }\end{array}$} & \multicolumn{22}{|c|}{ Zone of inhibition ( $\mathrm{mm}$ ) of fungi } \\
\hline & \multicolumn{22}{|c|}{ Candida albican } \\
\hline & S.no & 1 & 2 & 3 & 4 & 5 & 6 & 7 & 8 & 9 & 10 & 11 & 12 & 13 & 14 & 15 & 16 & 17 & 18 & 19 & 20 & Mean \\
\hline \multicolumn{2}{|c|}{ Ocimum basilicum } & 15 & 12 & 12 & 16 & 15 & - & 10 & - & 8 & 14 & 8 & 15 & 17 & 8 & 17 & 10 & - & 14 & 10 & 11 & 12.5 \\
\hline \multicolumn{2}{|c|}{ Cymbopogon citratus } & 14 & _ & - & 15 & 16 & 10 & _ & 8 & 13 & 14 & 7 & 14 & 18 & 9 & 16 & 10 & 8 & 15 & 12 & - & 12.3 \\
\hline \multicolumn{2}{|c|}{ Olea europaea } & 25 & 24 & 25 & 26 & 24 & 25 & 28 & 24 & 27 & 26 & 30 & 23 & 25 & 28 & 24 & 23 & 22 & 24 & 23 & 22 & 25.6 \\
\hline \multicolumn{2}{|c|}{ Eucalyptus camaldulensis } & 22 & 15 & 18 & 17 & - & 15 & 16 & 20 & 22 & 16 & - & 5 & 14 & 15 & 22 & 24 & 18 & 20 & 14 & 15 & 13 \\
\hline
\end{tabular}

*_: No activity

Table 5: Antimicrobial susceptibility of selected 01 fungal strain (Candida albican) against 50\% hydro alcoholic extract of leaves of medicinal plant. 
Citation: Zehra A, Naqvi SBS, Ali SQ (2016) In Vitro Evaluation of Antimicrobial Effect of Extracts of Medicinal Plant's Leaves. J Med Microb Diagn 5: 236. doi:10.4172/2161-0703.1000236

Page 9 of 14

\begin{tabular}{|c|c|c|c|c|c|c|c|c|c|}
\hline \multicolumn{10}{|c|}{ Zone of Inhibition ( $\mathrm{mm}$ ) of Positive and Negative Control Against tested clinical isolates } \\
\hline \multicolumn{2}{|c|}{ Micro-organism } & \multicolumn{4}{|c|}{ Gram Positive Bacteria } & \multicolumn{4}{|c|}{ Gram Negative Bacteria } \\
\hline \multicolumn{2}{|c|}{ Clinical Isolates } & & & & & & & & \\
\hline S.No & $\begin{array}{c}\text { Positive control - } \\
\text { Bacteria }\end{array}$ & $\begin{array}{c}\text { Staphylococcus } \\
\text { aureus }\end{array}$ & $\begin{array}{l}\text { Bacillus } \\
\text { subtilis }\end{array}$ & $\begin{array}{c}\text { Staphylococcus } \\
\text { epidermidis }\end{array}$ & $\begin{array}{l}\text { Enterococcus } \\
\text { faecalis }\end{array}$ & Escherichia coli & $\begin{array}{c}\text { Klebsiella } \\
\text { pneumonae }\end{array}$ & $\begin{array}{c}\text { Pseudomonas } \\
\text { aeruginosa }\end{array}$ & $\begin{array}{c}\text { Salmonella } \\
\text { typhi }\end{array}$ \\
\hline 1 & Ciprofloxacin $5 \mu \mathrm{g}$ & 21 & 19 & 27 & 35 & 22 & 20 & 18 & 22 \\
\hline 2 & Ciprofloxacin $5 \mu \mathrm{g}$ & 35 & 20 & 17 & 19 & 24 & 18 & 19 & 19 \\
\hline 3 & Ciprofloxacin $5 \mu \mathrm{g}$ & 18 & 14 & 25 & 21 & 19 & 21 & 21 & 22 \\
\hline 4 & Ciprofloxacin $5 \mu \mathrm{g}$ & 35 & 21 & 18 & 18 & 20 & 19 & 21 & 22 \\
\hline 5 & Ciprofloxacin $5 \mu \mathrm{g}$ & 37 & 19 & 26 & 24 & 22 & 20 & 37 & 22 \\
\hline 6 & Ciprofloxacin $5 \mu \mathrm{g}$ & 23 & 20 & 22 & 23 & 22 & 14 & 30 & 21 \\
\hline 7 & Ciprofloxacin $5 \mu \mathrm{g}$ & 25 & 27 & 23 & 11 & 22 & 21 & 35 & 20 \\
\hline 8 & Ciprofloxacin $5 \mu \mathrm{g}$ & 21 & 23 & 30 & 22 & 18.5 & 19 & 18 & 23 \\
\hline 9 & Ciprofloxacin $5 \mu \mathrm{g}$ & 22 & 11 & 25 & 23 & 19.5 & 20 & 22 & 22 \\
\hline 10 & Ciprofloxacin $5 \mu \mathrm{g}$ & 24 & 22 & 18 & 23 & 21 & 27 & 30 & 27 \\
\hline 11 & Ciprofloxacin $5 \mu \mathrm{g}$ & 23 & 24 & 22 & 30 & 18.5 & 20 & 30 & 23 \\
\hline 12 & Ciprofloxacin $5 \mu \mathrm{g}$ & 21 & 19 & 15 & 20 & 22 & 19 & 40 & 22 \\
\hline 13 & Ciprofloxacin $5 \mu \mathrm{g}$ & 26 & 20 & 22 & 21 & 19 & 20 & 19 & 20 \\
\hline 14 & Ciprofloxacin $5 \mu \mathrm{g}$ & 20 & 22 & 20 & 20 & 24 & 19 & 20 & 22 \\
\hline 15 & Ciprofloxacin $5 \mu \mathrm{g}$ & 23 & 22 & 21 & 21 & 25 & 22 & 16 & 21 \\
\hline 16 & Ciprofloxacin $5 \mu \mathrm{g}$ & 25 & 22 & 31 & 18 & 29 & 21 & 16 & 22 \\
\hline 17 & Ciprofloxacin $5 \mu \mathrm{g}$ & 30 & 18.5 & 26 & 25 & 24 & 20 & 19 & 20 \\
\hline 18 & Ciprofloxacin $5 \mu \mathrm{g}$ & 14 & 22 & 22 & 22 & 21 & 19 & 13 & 22 \\
\hline 19 & Ciprofloxacin $5 \mu \mathrm{g}$ & 23 & 19 & 23 & 22 & 36 & 21 & 20 & 12 \\
\hline 20 & Ciprofloxacin $5 \mu \mathrm{g}$ & 22 & 24 & 20 & 11 & 11 & 22 & 19 & 22 \\
\hline Mean & Ciprofloxacin $5 \mu \mathrm{g}$ & 22.45 & 21 & 23.75 & 24 & 21.8 & 20.4 & 23.2 & 24.66 \\
\hline \multicolumn{2}{|c|}{ Positive control - Fungai } & \multicolumn{3}{|c|}{ Candida albican } & \multicolumn{5}{|c|}{ Negative Control } \\
\hline 1 & Fluconazole $10 \mu \mathrm{g}$ & \multicolumn{3}{|c|}{20} & \multicolumn{3}{|c|}{ Hydro alcoholic Solvent } & \multicolumn{2}{|l|}{${ }^{*}$} \\
\hline 2 & Fluconazole $10 \mu \mathrm{g}$ & \multicolumn{3}{|c|}{22} & \multicolumn{3}{|c|}{ Hydro alcoholic Solvent } & \multicolumn{2}{|l|}{ - } \\
\hline 3 & Fluconazole $10 \mu \mathrm{g}$ & \multicolumn{3}{|c|}{22} & \multicolumn{3}{|c|}{ Hydro alcoholic Solvent } & \multicolumn{2}{|l|}{ - } \\
\hline 4 & Fluconazole $10 \mu \mathrm{g}$ & \multicolumn{3}{|c|}{30} & \multicolumn{3}{|c|}{ Hydro alcoholic Solvent } & \multicolumn{2}{|l|}{ - } \\
\hline 5 & Fluconazole $10 \mu \mathrm{g}$ & \multicolumn{3}{|c|}{23} & & Hydro alcoholic Solv & & - & \\
\hline 6 & Fluconazole $10 \mu \mathrm{g}$ & & 22 & & & Hydro alcoholic Solv & & - & \\
\hline 7 & Fluconazole $10 \mu \mathrm{g}$ & & 20 & & & Hydro alcoholic Solv & & - & \\
\hline 8 & Fluconazole $10 \mu \mathrm{g}$ & & 18 & & & Hydro alcoholic Solv & & - & \\
\hline 9 & Fluconazole $10 \mu \mathrm{g}$ & & 30 & & & Hydro alcoholic Solv & & _ & \\
\hline 10 & Fluconazole $10 \mu \mathrm{g}$ & & 31 & & & Hydro alcoholic Solv & & - & \\
\hline 11 & Fluconazole $10 \mu \mathrm{g}$ & & 22 & & & Hydro alcoholic Solv & & - & \\
\hline 12 & Fluconazole $10 \mu \mathrm{g}$ & & 20 & & & Hydro alcoholic Solv & & - & \\
\hline 13 & Fluconazole $10 \mu \mathrm{g}$ & & 24 & & & Hydro alcoholic Solv & & - & \\
\hline 14 & Fluconazole $10 \mu \mathrm{g}$ & & 24 & & & Hydro alcoholic Solv & & - & \\
\hline 15 & Fluconazole $10 \mu \mathrm{g}$ & & 22 & & & Hydro alcoholic Solv & & - & \\
\hline 16 & Fluconazole $10 \mu \mathrm{g}$ & & 25 & & & Hydro alcoholic Solv & & - & \\
\hline 17 & Fluconazole $10 \mu \mathrm{g}$ & & 25 & & & Hydro alcoholic Solv & & - & \\
\hline 18 & Fluconazole $10 \mu \mathrm{g}$ & & 22 & & & Hydro alcoholic Solv & & - & \\
\hline 19 & Fluconazole $10 \mu \mathrm{g}$ & & 30 & & & Hydro alcoholic Solv & & - & \\
\hline 20 & Fluconazole $10 \mu \mathrm{g}$ & & 32 & & & Hydro alcoholic Solv & & - & \\
\hline
\end{tabular}

Table 6: Antimicrobial susceptibility of selected studied microbes against positive and negative control.

\begin{tabular}{|c|c|c|c|c|c|}
\hline & \multirow{2}{*}{ Organism Type } & \multicolumn{4}{|c|}{ Diameter of zone of inhibition $(\mathrm{mm})$ Mean values of plants } \\
\hline & & Ocimum basilicum & Cymbopogon citratuss & Olea europaea & Eucalyptus camaldulens \\
\hline \multirow{4}{*}{$\begin{array}{c}\text { Gram Positive } \\
\text { Bacteria }\end{array}$} & Enterococcus faecalis & 3 & 12 & 25 & 5 \\
\hline & Bacillus subtilis. & 19 & 13.5 & 20 & 8 \\
\hline & Staphylococcus aureus & 12.8 & 14 & 25 & 12 \\
\hline & Staphylococcus epidermidis & 8 & 8 & 12 & 25 \\
\hline \multirow{4}{*}{$\begin{array}{c}\text { Gram } \\
\text { Negative } \\
\text { Bacteria }\end{array}$} & Escherichia coli & 4 & 14 & 22 & 20 \\
\hline & Klebsiella pneumonae & 12 & 8 & 30 & 10 \\
\hline & Pseudomonas aeruginosa & 4.5 & 6.5 & 18 & 10 \\
\hline & Salmonella typhi & 25 & 20 & 30 & 25 \\
\hline Fungai & Candida albicans & 12.5 & 12.3 & 25.6 & 13 \\
\hline
\end{tabular}

Table 7: Mean Value of antimicrobial susceptibility of selected microbes against $50 \%$ hydro alcoholic extract of leaves of medicinal plant. 


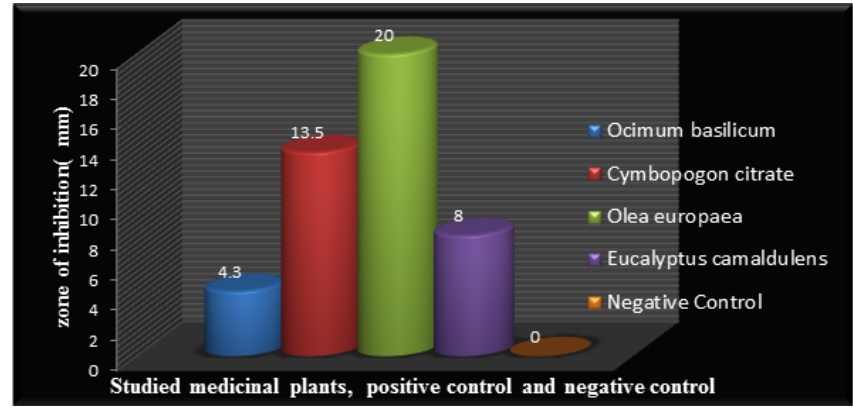

Figure 13: Antimicrobial sensitivity pattern of Bacillus subtilis against studied medicinal plants, positive control (ciprofloxacin) and negative control $(50 \%$ hydroalcoholic solvent).

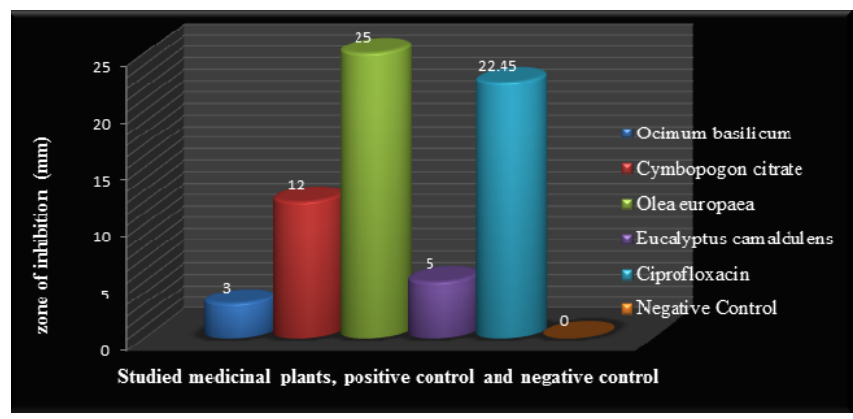

Figure 14: Antimicrobial sensitivity pattern of Staphylococcus aureus against studied medicinal plants, positive control (ciprofloxacin) and negative control ( $50 \%$ hydroalcoholic solvent).

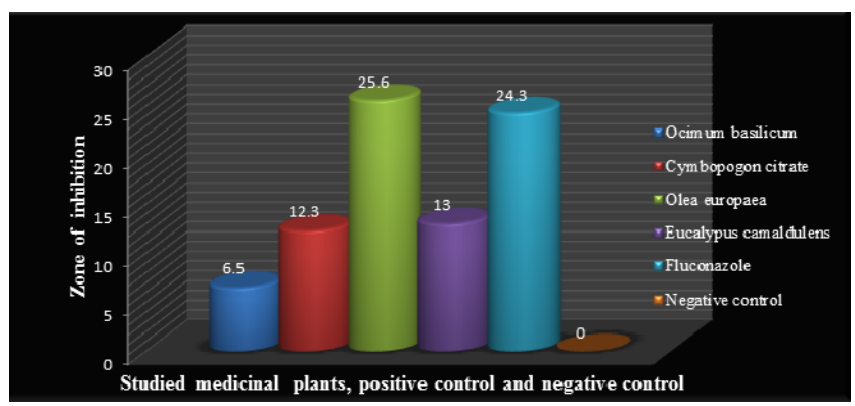

Figure 15: Antimicrobial sensitivity pattern of Candida albican against studied medicinal plants, positive control (Fluconazole) and negative control (50\% hydroalcoholic solvent).

susceptibility to Olive leaf extract in current study, and showed a zone of inhibition of $20 \mathrm{~mm}$ as shown in Figure 13.

Mihriban et al. reported [73] antimicrobial effect of extract of olive leaf and proved its activity against Staphylococcus, Salmonella, Klebsiella and Enterococcus species. Similarly Hawn and Yong [74] also reported its antimicrobial activity.

S. aureus showed a zone of inhibition of average $25 \mathrm{~mm}$ in current study as shown in Figure14. Belgizar and Nazan worked on olive leaves based extract and reported to be effected as antimicrobial.

Least activity was observed on S. epidermidis i.e. $12 \mathrm{~mm}$ as shown in Table 4. C. albican showed greater zone of inhibition $(25.6 \mathrm{~mm}$; Table 7) in current study against olive leaves extract as compare to other extract of medicinal plant used during the study.

S. typhi and K. pneumonae showed a marked increase in susceptibility $30 \mathrm{~mm}$ and $30 \mathrm{~mm}$ respectively (Table 7) which were greater as compared to positive control ciprofloxacin $24.6 \mathrm{~mm}$ and $20.4 \mathrm{~mm}$ respectively as shown in Table 6. Hwan and Yong [74] also reported antibacterial activity of extract of olive leaf.

\section{Ocimum basilicum}

O. basilicum L. has been used traditionally as an herbal medicine since ancient period of time [81-84].

Invitro antimicrobial effect of extract of leaves of O. basilicum L. was assessed by [85] and reported the antimicrobial activity of $\mathrm{O}$. basilicum L. [86].

Among selected Gram positive bacteria, S. aureus showed larger zone of inhibition $12.8 \mathrm{~mm}$ while E. faecalis showed least zone of inhibition $03 \mathrm{~mm}$ as shown in Table 7. B. subtilis play a vital role in skin disease. In current study the extract of leaves of O. basilicum L., B. subtilis showed a zone of inhibition of $19 \mathrm{~mm}$ as shown in Figure 13 while ciprofloxacin was used as positive control and showed a zone of inhibition of $21 \mathrm{~mm}$ as shown in Table 6. Budka and Khan [32] also reported the bactericidal activity of Bacillus spp. by extract of $\mathrm{O}$. basilicum $L$.

Similarly antibacterial activity of O. basilicum L. against Gram negative bacteria was higher against $\mathrm{S}$. typhi showed $25 \mathrm{~mm}$ zone of inhibition while $E$. coli showed a minimum zone of inhibition $4 \mathrm{~mm}$ as shown in Table 7 . However Tyagi et al. $[87,88]$ also reported the antibacterial activity of E. coli against O. basilicum L. Variation in result may be due to increase resistant pattern of microbes day by day.

Similarly Balamurugan [89] studied antibacterial activity of leaves of O. basilicum L. against B. subtilis, E. coli, S. typhi and on 02 fungal strains i.e. A. niger and C. albican. E. coli and C. albican showed good succeptibilty pattern against $\mathrm{O}$. bacilicum, Kaeidi et al. and Ito et al. [90,91], presented antimicrobial effect of extract of leaves of O. basilicum L. and found that due to presence of carbozole alkaloids, $\mathrm{O}$. basilicum L. possessed an antimicrobial activity [92].

Balamurugan reported [89] that C. albican showed a zone of inhibition of $14 \mathrm{~mm}$ in methanolic extract of O. basilicum L. In current study the selected fungal strain i.e. C. albican showed a zone of inhibition of $12.5 \mathrm{~mm}$ (Figure 15) which is almost same with the work of Balamurugan [89]. Broad spectrum antifungal, fluconazole showed a good sensitivity against C. albican, zone of inhibition was $24.3 \mathrm{~mm}$ as shown in Figures 12 and 15.

\section{Eucalytpus camaldulensis}

E. camaldulensis commonly known as river red gum commonly found in Pakistan. Its traditional medicinal usage is very old since ancient period of time. Extract of leaf of Red River gum showed mixed activity against studied microbes. Various studies have been done to support the traditional use of leaves of E. camaldulensis Dehnh. as antibacterial and antifungal [84,93-96].

Oyedeji et al. reported the [94] antibacterial activity of E. camaldulensis Dehnh. against Gram positive bacteria, Gram negative bacteria and anti-fungal activity against Candida albican [97].

S. epidermidis, among selected Gram positive bacteria showed a larger zone of inhibition $25 \mathrm{~mm}$ (Table 7) while E. faecalis shown a least zone of inhibition $5 \mathrm{~mm}$ when compared with broad spectrum antibiotic ciprofloxacin (Figure 16) indicate its poor sensitivity to extract of E. camaldulensis Dehnh. 
Sakaino et al. reported [97] antibacterial activity of Staphylococcus aureus. In current study, among Gram negative bacteria $S$. aureus showed an intermediate zone of inhibition $12 \mathrm{~mm}$ while E. coli showed a zone of inhibition of $20 \mathrm{~mm}$ (Figure 17).

Testunari showed antibacterial [98] and fungicidal activity of leaves of E. camaldulensis Dehnh. Against S. aureus, B. subtilis, E. coli and A. niger.

Extract of leaves of E. camaldulensis Dehnh. Showed antibacterial activity against Klebsiella spp., S. typhi, P. aeruginosa, S. aureus and B. subtilis by the agar diffusion method $[93,99]$.

Fungal activity of eucalyptus was least as shown in Table 6 while [100] worked on antifungal activity of E. camaldulensis Dehnh.

Kretsi et al. reported [101] antimicrobial effect of E. camaldulensis Dehnh. The difference may be due to increase resistance pattern day by day by microbes and depend on usage criteria from individuals of specific region and cultivation.

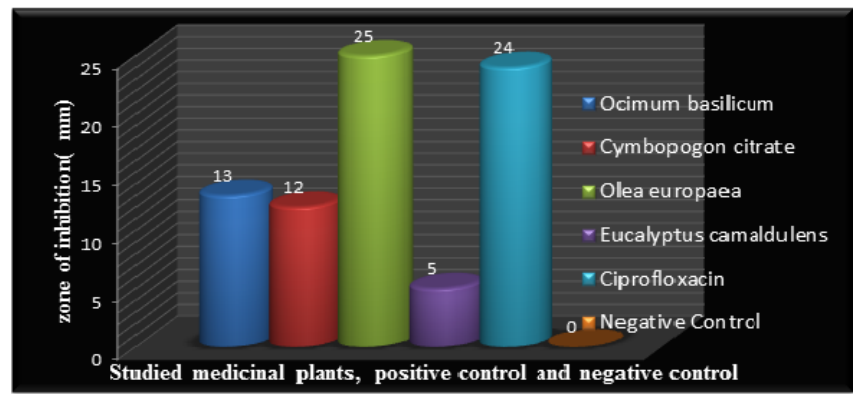

Figure 16: Antimicrobial sensitivity pattern of Enterococcus faecalis against studied medicinal plants, positive control (ciprofloxacin) and negative control (50\% hydroalcoholic solvent).

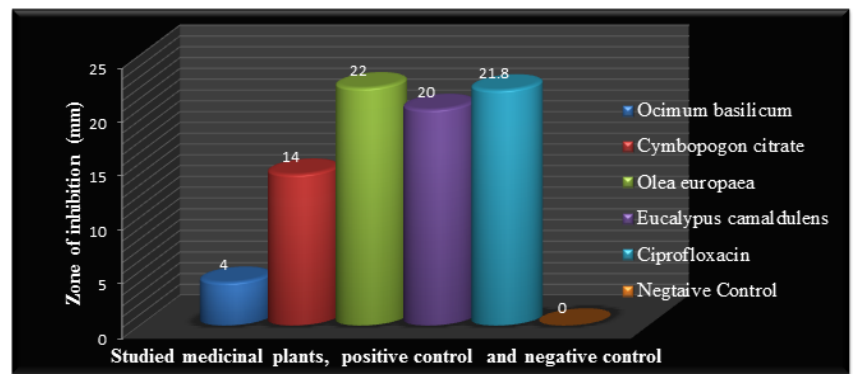

Figure 17: Antimicrobial sensitivity pattern of Escherichia coli against studied medicinal plants, positive control (ciprofloxacin) and negative control $(50 \%$ hydralcoholic solvent).

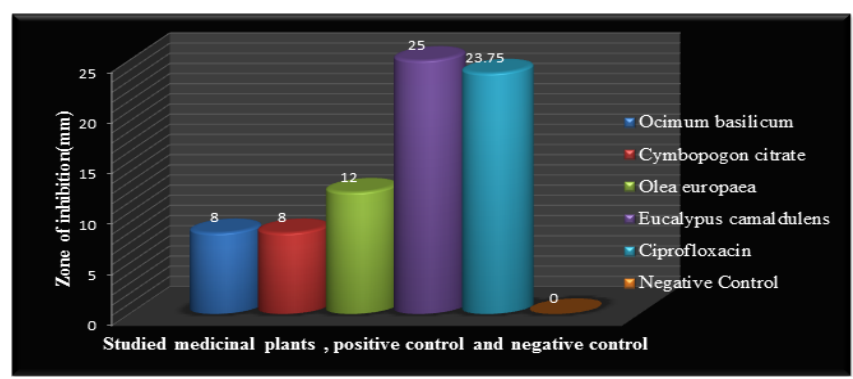

Figure 18: Antimicrobial sensitivity pattern of Staphylococcus epidermidis against studied medicinal plants, positive control (ciprofloxacin) and negative control ( $50 \%$ hydroalcoholic solvent).

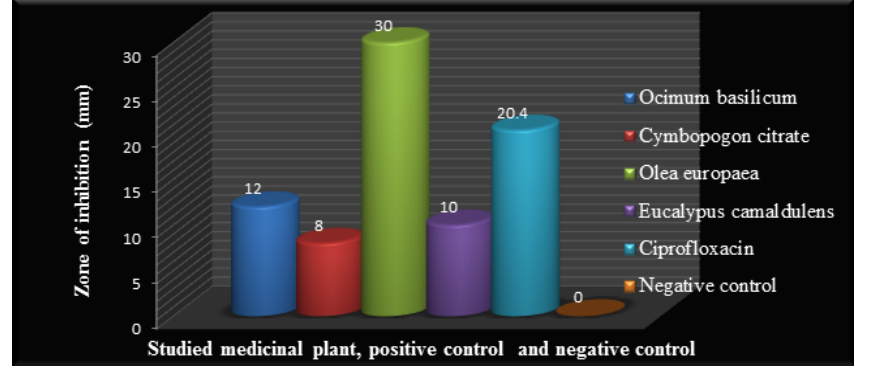

Figure 19: Antimicrobial sensitivity pattern of Klebsiella pneumonae against studied medicinal plants, positive control (ciprofloxacin) and negative control ( $50 \%$ hydroalcoholic solvent)

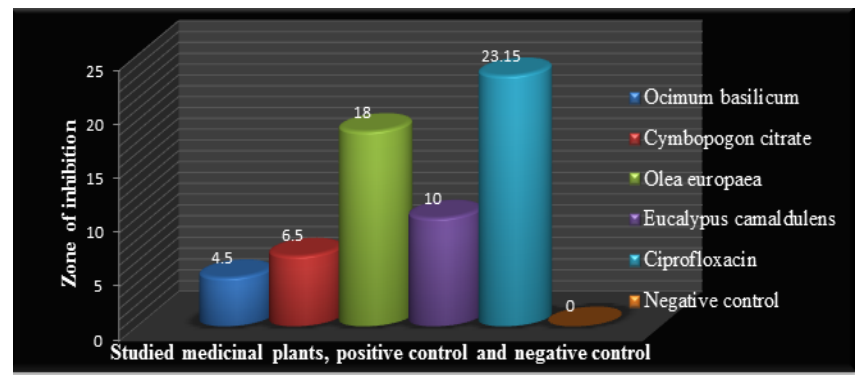

Figure 20: Antimicrobial sensitivity pattern of Pseudomonas aeruginosa against studied medicinal plants, positive control (ciprofloxacin) and negative control ( $50 \%$ hydroalcoholic solvent)

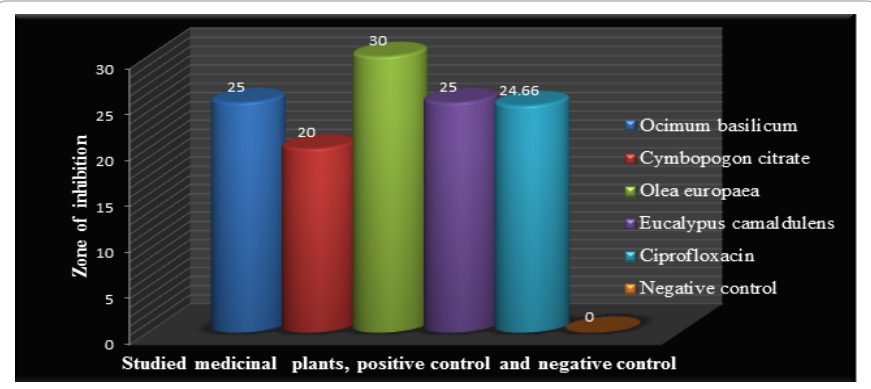

Figure 21: Antimicrobial sensitivity pattern of Salmonella typhi against studied medicinal plants, positive control (ciprofloxacin) and negative control $(50 \%$ hydroalcoholic solvent).

All extract showed good activity against broad spectrum antibiotic ciprofloxacin with different zone of variation. In this investigation, there is slight variation in zone of inhibition which may be neglected as mean value was calculated and this reasons supposed for variation of zones among the same pathogen even isolated from the same sources (Figures 18-21)

\section{Cymbopogon citratus}

Extract of C. citratus (DC.) Stapf. (Commonly known as Lemon grass) has been used as traditional medicines and extensive work has been done to prove its medicinal activity with verification of safety intake [102-107] investigated and reported that extract of leaves of C. citratus (DC.) Stapf. Contain antimicrobial activity against all microbes.

Tyagi and Malik worked on [87] sensitivity of one of fungal strain, Candida albican against extract of C. citratus. In current study Candida albican showed a zone of inhibition of $12.3 \mathrm{~mm}$ which was less when compared with positive control fluconazole (showed a zone of inhibiton of $24.3 \mathrm{~mm}$ ) as shown in Table 6 . Variation in zone may be due to resistant of microbe developed day by day. 
Adegoke and Odesola reported the [105] antimicrobial activity of C. citratus (DC.) Stapf. Against Gram positive bacteria; B. subtilis, S. aureus, Gram negative bacteria; E. coli, P. aeruginosa and few strain of fungi. S. typhi showed a greater zone of inhibition $20 \mathrm{~mm}$ as shown in Table 7 in current study among all Gram negative bacteria while B. subtilitis showed a greater zone of inhibition $13.5 \mathrm{~mm}$ as shown in Table 6 as compare to other selected Gram positive bacteria.

Similarly Mihriban et al. [73] worked on C. citratus (DC.) Stapf. Bactericidal activity against $S$. aureus, B. subtilis, E. coli, K. pneumoniae and P. aeruginos, using Agar Diffusion Method and Broth Dilution Method.

\section{Conclusion}

Overall results of current study reflect that herbal medicinal plant possessed antimicrobial activity against different clinical isolates. There is need to explore those plants. Among selected studied medicinal plant, O. europea leaf showed more antibacterial and antifungal activity.

\section{References}

1. Madigan M, Martinko J (2006) Brock Biology of Microorganisms. (13thedn), Pearson Education 1096

2. Donadio S, Maffioli S, Monciardini P, Sosio M, Jabes D (2010) Antibiotic discovery in the twenty-first century: Current trends and future perspectives, The J Antibio 63: 423-430.

3. Mothana RA, Lindequist $U$ (2005) Antimicrobial activity of some medicinal plants of the island Soqotra, J Ethnopharmacol 96: 177-181.

4. van Vuuren SF (2008) Antimicrobial activity of South African medicinal plants J Ethnopharmacol 119: 462-472.

5. Srinivasan D, Nathan S, Suresh T, Lakshmana Perumalsamy P (2001) Antimicrobial activity of certain Indian medicinal plants used in folkloric medicine J Ethnopharmacol 74: 217-220.

6. Sindambiwe JB, Calomme M, Cos P, Totté J, Pieters L, et al. (1999) Screening of seven selected Rwandan medicinal plants forantimicrobial and antiviral activities. J Ethnopharmacol 65: 71-77.

7. Jeevan RA, Bhakshu LMd, Venkata RRR (2004) In vitro antimicrobial activity of certain medicinal plants from Eastern Ghats, India, used for skin diseases. J Ethnopharmacol 90: 353-357.

8. Fabricant DS, Farnsworth NR (2001) The value of plants used in traditional medicine for drug discovery. Environ Health Perspect 109: 69-75.

9. John RS, Daniel EM (2001) The importance of weeds in ethnopharmacology. $J$ Ethnopharmacol 75: 19-23.

10. Pappas S (2009) Your Body Is a Wonderland of Bacteria. Science NOW Daily News.

11. Todar K (2011) Normal Bacterial Flora of Humans Todar's, Online Textbook of Bacteriology. 1-5.

12. Cogen AL, Nizet V, Gallo RL (2008) Skin microbiota: a source of disease or defence? Br J Dermato 158: 442-455.

13. Trent JT, Federman D, Kirsner RS (2001) Common bacterial skin infections, Ostomy Wound Manage 47: 30-34.

14. Uniyal SK, Singh KN, Jamwal P, Lal B (2006) Traditional use of medicinal plants among the tribal communities of Chhota Bhangal, Western Himalayan. J. Ethnobiol Ethnomed $2: 1$

15. Balandrin MF, Klocke JA, Wurtele ES, Bollinger WH (1985) Wurtele and W.H. Bollinger, Natural plant chemicals Sources of Industrial and Medicinal materials. Science 228: 1154-1160.

16. Samy RP, Ignacimuthu S (2000) Antibacterial activity of some folklore medicinal plants used by tribals in Western Ghats in India. J Ethnopharmacol 69: 63-71.

17. Kumarasamy Y, Cox PJ, Jaspars M, Nahar L, Sarker SD (2002) Screening seeds of Scottish plants for antibacterial activity. J Ethnopharmacol 83: 73-77.

18. Stepanović S1, Antić N, Dakić I, Svabić-Vlahović M (2003) In vitro antimicrobail activity of propilis and antimicrobial drug. Microbial Resis 158:353-357.
19. Chiang LC, Ng LT, Cheng PW, Chiang W, Lin CC (2005) Antiviral activities of extracts and selected pure constituents of Ocimum basilicum. Clin Exp Pharmacol Physiol 32: 811-860.

20. Manosroi J, Dhumtanom P, Manosroi A (2006) Anti-proliferative activity of essential oil extracted from Thai medicinal plants on KB and P388 cell lines. Cancer Lett 235: 114-120.

21. Blanco MM, Costa CA, Freire AO, Santos JG, Costa M (2009) Neurobehaviora effect of essential oil of Cymbopogon citratus in mice. Phytomedicine 16: 265-70.

22. Francois P, Schrenzel J (2008) "Rapid Diagnosis and Typing of Staphylococcus aureus". Staphylococcus: Molecular Genetics. Caister Academic Press.

23. Murray PR, Baron E J, Jorgensen JH, Landry ML, Pfaller MA, et al. (Eds.) (2003) Manual of Clinical Microbiology (8thedn), Herdon, VA, United States of America: American Society for Microbiology.

24. Eisenstein BI (2008) Treatment challenges in the management of complicated skin and soft-tissue infections. Clinical Microbiology and Infection. Clin Microbiol Infect 14: 17-25.

25. Fridkin SK, Hageman JC, Morrison M, Sanza LT, Como-Sabetti K, et al. (2005) Methicillin-resistant Staphylococcus aureus disease in three communities. N Engl J Med 352: 1436-1444.

26. Somova LI, Shode FO, Ramnanan P, Nadar A (2003) Antihypertensive antiatherosclerotic and antioxidant activity of triterpenoids isolated from Olea europaea, subspecies africana leaves. J Ethnopharmacol 84: 299-305.

27. Miller LG, Perdreau-Remington F, Rieg G, Mehdi S, Perlroth J, et al. (2005) Necrotizing fasciitis caused by community-associated methicillin-resistant Staphylococcus aureus in Los Angeles. N Engl J Med 352: 1445-1453.

28. Zaidi TS, Priebe GP, Pier GB (2006) A live-attenuated Pseudomonas aeruginosa vaccine elicits outer membrane protein-specific active and passive protection against corneal infection. Infect Immun 74: 975-983.

29. Pruitt BA, McManus AT, Kim SH, Goodwin CW (1998) Burn wound infections: current status. World J Surg 22:135-145.

30. Hayley B (2008) Anti-inflammatory compound from cannabis found in herbs, RSC advancing the chemical sciences.

31. Botsoglou E, Govaris A, Fletouris D, lliadis S (2013) Olive leaves (Olea europea) and -tocopheryl acetate as feed antioxidants for improving the oxidative stability of -linolenic acid-enriched eggs. J Anim Physiol Anim Nutr 97: 740-753.

32. Budka D, Khan N A (2010) The Effect of Ocimum basilicum, Thymus vulgaris, Origanum vulgare Essential Oils on Bacillus cereus in Rice-Based Foods. EJBS 2.

33. Lewinsohn E, Ziv-Raz I, Dudai N, Tadmor $Y$, Lastochkin E, et al. (2000) Biosynthesis of estragole and methyl-eugenol in sweet basil (Ocimum basilicum L). Developmental and chemotypic association of allylpheno O-methyltransferase activities. Plant Sci 160: 27-35.

34. de Almeida I, Alviano DS, Vieira DP, Alves PB, Blank AF, et al. (2007) Antigiardial activity of Ocimum basilicum essential oil. Parasitol Res 101: 443-452.

35. Tohti I, Tursun M, Umar A, Turdi S, Imin H, et al. (2006) Aqueous extracts of Ocimum basilicum (sweet basil) decrease platelet aggregation induced by ADP and thrombin in vitro and rats arterio--venous shunt thrombosis in vivo, Thromb 118: 733-739.

36. Shadab Q, Hanif M, Chaudhary FM (1992) Antifungal activity by lemongrass essential oils. Pak. J Sci Ind Res 35: 246-249.

37. Wang L, Waltenberger B, Pferschy-Wenzig EM, Blunder M, Liu X, et al. (2014) Natural product agonists of peroxisome proliferator-activated receptor gamma (PPARy): a review. Biochem Pharmacol 92: 73-89.

38. Sakaria Baby P, Joy PP, Mathew S, Mathew G, Joseph A, et al. (2007) Aromatic Plants. 1: 103.

39. Micol V, Caturla N, Pérez-Fons L, Más V, Pérez L, et al. (2005) The olive leaf extract exhibits antiviral activity against viral haemorrhagic septicaemia rhabdovirus (VHSV). Antiviral Res 66: 129-136.

40. Kayser FH, Bienz KA, Eckert J, Zingernagel RM (Eds.) (2001) Medical Microbiology (10thedn; Stuttgart, Germany: Georg Thieme Verlag.

41. Shellito J, Nelson S, Sorensen RU (1992) Effect of pyocyanine, a pigment of 
Pseudomonas aeruginosa, on production of reactive nitrogen intermediates by murine alveolar macrophages. Infect Immun 60: 3913-3915

42. Begum S, Sultana I, Siddiqui BS, Shaheen F, Gilani AH (2002) Structure and spasmolytic activity of eucalyptanoic acid from Eucalyptus camaldulensis var obtusa and synthesis of its active derivative from oleanolic acid. J Nat Prod 65: 1939-1941.

43. Ayana B, Turhan KN (2009) Use of antimicrobial methylcellulose films to control Staphylococcus aureus during storage of Kasar cheese. Packaging Technology \& Science 22: 461-469.

44. Willcox M D (2007) Pseudomonas aeruginosa infection and inflammation during contact lens wear: a review. Optom Vis Sci 84: 273-278.

45. Hussain GA, Arif ullah K, Muhammad GN (2006) Ca2+ antagonist and cholinergic activities explain the medicinal use of olive in gut disorders. Nutr Res 26: 277-283.

46. Khayyal MT, el-Ghazaly MA, Abdallah DM, Nassar NN, Okpanyi SN, et al. (2002) Blood pressure lowering eff-ect of an olive leaf extract (Olea europaea) in L-NAME induced hypertension in rats. Arzneimittelforschung 52: 797-802.

47. Benavente GO, Castillo J, Lorente J, Ortuño A, del Rio JA (2000) Antioxidan activity of phenols extracted from Olea europaea leaves, Food Chemistry 68: 457-462.

48. BIO 347 Laboratories 15 and 16 Antimicrobial Susceptibility Testing 73-80.

49. Watt JM, Breyer-Brandwijk MG (1962) The medicinal and poisonous plants of southern and eastern Africa. (2ndedn) E. \&S. Livingstone, Ltd., Edinburgh and London.

50. Zarzuelo A, Duarte J, Jimenez J, Gonzalez M, Utrilla MP (1991) Vasodilator effect of olive leaf. Planta Med 57: 417-9.

51. Palumbo SA (1972) Role of iron and sulfur in pigment and slime formation by Pseudomonas aeruginosa. J Bacteriol 111: 430-436.

52. Abuismail M (2011) Analysis and Biological Evaluation of Jordanian Olive Trees Leaves-Cancer Cure Invention with Tissue Repair Potential, VDM VERLAG DR. MÜLLER. Besorgungstitel-vorauss. Lieferzeit 3-5.

53. Stevenson L (2006) In vitro Biological Activities of Pure Olive Leaf Extract \& High Strength Olive Leaf Extract. Ameriden International Inc.

54. Hamdi $\mathrm{O}$ (2005) A non-toxic olive iridoid, is an anti-tumor agent and cytoskeleton disruptor. Biochem Biophys Res Commun 334: 769-778.

55. Pinelli P, Carlotta G, Mulinacci N, Vincieri F, Tattini M, et al. (2000) Qualiquantitative analysis and antioxidant activity of different polyphenolic extracts from Olea europea L. leaves. J commodity sci, Source 39: 71-83.

56. Antonella S, Domenico T, Antonio T, Rossella Lo, Cascio PP, et al. (1998) In vitro evaluation of the antioxidant activity and Biomembrane interaction of the plant phenols oleuropein and hydroxytyrosol. Int J Pharm 166: 123-133.

57. Speroni, E, Guerra MC, Minghetti A, Crespi-Perellino N, Pasini P, et al. (1998) Oleuropein Evaluated In Vitro and In Vivo as an Antioxidant. Phytother Res 12

58. James DA (1981) The gene revolution.1981b: 89-150.

59. El-Mahmood MA (2010) Antibacterial potential of crude leaf extracts of Eucalyptus camaldulensis against some pathogenic bacteria. AJPS 4: 202-209.

60. Perez C, Paul M, Bazerque P (1990) An antibiotic assay by the agar well diffusion method. Acta Biol Med Exp 15: 113-115.

61. Nair R, Chanda S (2007) In vitro antimicrobial activity of Psidium guajava leaf extracts against clinically important pathogenic microbial strain. Braz J Micro 38: $452-458$

62. Achinto S, Kawshik KC, Sitest CB, Surash CR, Joydeb KK (2005) Antiinflammatory, analgesic and diuretic activity of PohygorumLanatum Roxh. Pak. L J Pharma Sci 8: 13-18.

63. Bauer AW, Kirby WMM, Sherris JC, Turck M (1966) Antibiotic susceptibility testing by a standardized single disk method. Am J Clin Pathol 45: 493-496.

64. Parekh J, Chanda S (2006) In vitro antimicrobial activities of extracts of Launaea procumbens Roxb. (Labiateae), Vitis vinifera L. (Vitaceae) and Cyperus rotundus L. (Cyperaceae). Afr J Biomed Res 9: 89-93.

65. Jha RK, Garud N, Nema RK (2009) Excision and Incision Wound Healing Activity of Flower Head Alcoholic Extract of Sphaeranthus indicus Linn. In Albino Rats. GJP 3: 32-37.
66. Huu T, Martin L (2014) Composition with extracts from olive leaf, yarrow and rosemary for treating human diseases and conditions. PCT Int. Appl.

67. Ersalan M (2014) Usage of Figure fruit extract and olive leaf extract combinations together with green tea and grape seed extracts for eliminating and preventing the formation of cancer cells. PCT Int. Appl.

68. Irfan NM, Bashir A, Fomda E, Javid A, Bhat, A (2010) Activity of lemongrass (Cymbopogon citratus) oil against some selected pathogenic bacterias. Asian Pac J Trop Med 3: 535-538.

69. Keceli TM, Harp F (2014) The effect of olive leaves and their harvest time on radical scavenging activity and oxidative stability of refined olive oil. QUAL ASSUR SAF CROP 6: 141-149.

70. Andreadou I, Mikros E, loannidis K, Sigala F, Naka K, et al. (2014) Oleuropein prevents doxorubicin-induced cardiomyopathy interfering with signaling molecules and cardiomyocyte metabolism. J Mol Cell Cardiol 69: 4-16.

71. Cabarkapa A, Zivkovic L, Zukovec D, Djelic N, Bajic V, et al. (2014) Protective effect of dry olive leaf extract in adrenaline induced DNA damage evaluated using in vitro comet assay with human peripheral leukocytes. Toxicol In Vitro 28: 451-456.

72. Shengzhao G (2013) A natural preservative composition and its application in cosmetics. Faming Zhuanli Shenqing.

73. Mihriban K, Sahan Y, Yigit A, Ozer ET, Gucer S (2010) Antibacterial activity and chemical constitutions of Olea europaea leaf extracts. J Food Process Preserv 34: 383-396.

74. Hwan Ok, Yong L (2010) Antioxidant and antimicrobial activities of individualand combined phenolics in Olea europaea leaf extract. Bioresour Technol 101: 3751-3754.

75. Belgizar A, Nazan TK (2009) Use of antimicrobial methylcellulose films to control Staphylococcus aureus during storage of Kasar cheese. Packaging Technology \& Science 22: 461-469.

76. Fernando FD (2012) Process for preparing a cosmetic composition comprising vitamins and plant extracts having an immediate lifting effect. Eur Pat Appl.

77. Hayes JE, Allen P, Brunton N, Grady MN, Kerry JP (2011) Phenolic composition and in vitro antioxidant capacity of four commercial phytochemical products: Olive leaf extract (Olea europaea), lutein, sesamol and ellagic acid. Food Chemistry 126: 948-955.

78. Eidi A, Eidi M, Darzi R (2009) Antidiabetic effect of Olea europaea in norma and diabetic rats. Phytother Res 23: 347-350.

79. Sudjana AN, D'Orazio C, Ryan V, Rasool N, Ng J, et al. (2009) Antimicrobial activity of commercial Olea europaea (olive) leafextract. Int $\mathrm{J}$ Antimicrob Agents 33: 461-463.

80. Susalit E, Agus N, Effendi I, Tjandrawinata RR, Nofiarny D, et al. (2011) Olive (Olea europaea) leafextract effective in patients with stage-1 hypertension: comparison with Captopril. Phytomedicine 18: 251-258

81. Mohamed BIF, Hedya J, Mohamed A, Sami S (2008) Effect of storage on refined and husk olive oils composition: Stabilization by addition of natural antioxidants from Chemlali olive leaves. Food Chem 108: 253-262.

82. Vieira RF, Simon JE (2000) Chemical Characterization of Basil (Ocimum spp.) Found in the Markets and Used in Traditional Medicine in Brazil. Economic Botany 54: 207-216.

83. Dasgupta T, Rao AR, Yadava PK (2004) Chemomodulatory efficacy of Basi leaf (Ocimum basilicum) on drug metabolizing and antioxidant enzymes and on carcinogen-induced skin and forestomach papillomagenesis. Phytomedicine 11: $139-151$.

84. Grayer RJ, Kite GC, Goldstone FJ, Bryan SE, Paton A (1996) Infraspecific taxonomy and essential oil chemotypes in sweet basil, Ocimum basilicum Original Research Article Phytochemistry 43: 1033-1039.

85. Kristinsson KG, Magnusdottir AB, Petersen H, Hermansson A (2005) Effective Treatment of Experimental Acute Otitis Media by Application of Volatile Fluids into the Ear Canal. J Infect Dis 191: 1876-1880.

86. Opalchenova G, Obreshkova D (2003) Comparative studies on the activity of basil-an essential oil from Ocimum basilicum-against multidrug resistant clinical isolates of the genera Staphylococcus, Enterococcus and Pseudomonas by using different test methods. Original Research Article. J Microbiol Meth 54 105-110. 
87. Amit Kumar T, Anushree M (2010) In situ SEM, TEM and AFM studies of the antimicrobial activity of lemon grass oil in liquid and vapour phase against Candida albicans. Micron 41: 797-805.

88. Amit TK, Anushree M (2011) Bactericidal action of lemon grass oil vapours and negative air ions. Original Research Article. Innovative Food Science \& Emerging Technologies, In Press, Accepted Manuscript, Available online 2.

89. Balamurugan S (2013) In vitro antimicrobial activity of Ocimum basilicum Linn Leaf Extracts. Int J Recent Sci Res 4: 38-40.

90. Kaeidi A, Mahani ES, Vahid S, Mehdi A, Bahram R, et al. (2011) Olive (Olea europaea ) leaf extract attenuates early diabetic neuropathic pain through prevention of high glucose-induced apoptosis: In vitro and in vivo studies. J Ethnopharmacol 136: 188-196.

91. Ito C, Thoyama Y, Omra M, Ichiro K, Furukawa H (1933) Alkaloidal constituents of Ocimum basilicum:isolation and structural elucidation of novel binary carazolequinones and carazole alkaloids. chem Pharm Bull 41: 2096-2100.

92. Kum C, Kirkan S, Sekkin S, Akar F, Boyacioglu M (2008) Comparison of in vitro antimicrobial susceptibility in Flavobacterium psychrophilum isolated from rainbow trout fry. J Aquat Anim Health 20: 245-51.

93. Akin M, Aktumsek A, Nostro A (2010) Antibacterial activity and composition of the essential oils of Eucalyptus camaldulensis Dehn. and Myrtus communis L. growing in Northern Cyprus. AJB 9: 531-535

94. Oyedeji Adebola O, Ekundayo, Olusegun, Olawore, Olayide N, et al. (2000) Essential oil composition of two varieties of Eucalyptus camaldulensis Dehn. from Nigeria. JEOR 12: 102-104.

95. Oyedeji AO, Ekundayo O, Olawore, ON, Adeniyi BA, Koenig Wilfried A (1999) Antimicrobial activity of the essential oils of five Eucalyptus species growing in Nigeria. Fitoterapia 70: 526-528.

96. Chaudhari DC, Suri RK (1991) Comparative studies on chemical and antimicrobial activities of fast growing Eucalyptus hybrid (FRI-4 and FRI-5) with their parents. Minor For. Prod. Div., For. Res. Inst., Dehra Dun, India. Indian Perfumer 35 : $30-4$

97. Makoto S, Tetsunari T, Isao K (1999) Eucalyptus extracts as antibacterial agents. (Oji Paper Co., Ltd., Japan). Jpn. Kokai Tokkyo Koho 10:77.
98. Takahashi T. Microbicides containing Eucalyptus leaf extracts and chitosan. (Oji Paper Co., Ltd., Japan). Jpn. Kokai Tokkyo Koho, 19

99. Ayepola OO, Adeniyi BA (2008) The antibacterial activity of leaf extracts of Eucalyptus camaldulensis (Myrtaceae). J appl sci res 4: 1410-1413.

100. Chang YS, Lung CH, Eugene ICW, Chang ST (2006) Antifungal activities and chemical compositions of essential oils from leaves of four eucalypts. Taiwan Linye Ke xue, 21: 49-61.

101. Harvala E, Kretsi O, Chinou IB (2002) Composition and antibacterial activity of the essential oils of Eucalyptus camaldulensis growing in Greece. Dept. Pharmacognosy, Sch. Pharmacy, Univ. Athens, Athens, Greece. Proceedings of the Phytochemical Society of Europe 235-239.

102. Costa CA, Bidinotto LT, Takahira RK, Salvadori DM, Barbisan LF, et al. (2011) Cholesterol reduction and lack of genotoxic or toxic effects in mice after repeated 21-day oral intake of lemongrass (Cymbopogon citratus) essential oil. Food Chem Toxicol 49: 2268-2272.

103. Francisco V, Figueirinha A, Miguel Neves B, García-Rodríguez C, Celeste Lopes M (2011) Cymbopogon citratus as source of new and safe antiinflammatory drugs: Bio-guided assay using lipopolysaccharide-stimulated macrophages. J Ethnopharmacol 133: 818-827.

104. Adejuwon AA, Agbaje EO (2007) Hypoglycemic and hypolipidemic effects of fresh leaf aqueous extract of Cymbopogon citratus Stapf. in rats. J Ethnopharmacol 112: 440-444.

105. Adegoke GO, Odesola BA (1996) Storage of maize and cowpea and inhibition of microbial agents of biodeterioration using the powder and essential oil of lemon grass (Cymbopogon citratus). Original Research Article International Biodeterioration \& Biodegradation 37: 81-84.

106. Souza Formigoni ML, Lodder HM, Gianotti Filho O, Ferreira TM, Carlini EA (1986) Pharmacology of lemongrass (Cymbopogon citratus Stapf). II. Effects of daily two month administration in male and female rats and in offspring exposed "in utero". J Ethnopharmacol 17: 65-74.

107. Bassolé IH, Lamien-Meda A, Bayala B, Obame LC, Ilboudo AJ, et al. (2011) Chemical composition and antimicrobial activity of Cymbopogon citratus and Cymbopogon giganteus essential oils alone and in combination. Phytomedicine 18: 1070-1074. 